\title{
Efficient Reacquire and Identify Path Planning Over Large Areas
}

Abhishek Sriraman

\begin{abstract}
An important task in maritime search and inspection involves re-acquiring and identifying underwater objects by surveying the objects from multiple angles. Because of false contacts related to clutter on the sea floor, the objects are often detected in dramatically different densities in a given area. Previously developed methods to plan survey paths on groups of contacts led to efficient paths when the contacts occur in close proximity, but inefficient paths when the objects occur over large distances. We present a planning algorithm to generate an efficient path to survey objects from multiple angles that is independent of the density of the objects. The algorithm leverages the previously-developed algorithms for surveying objects from multiple directions, coupled with density-based spatial clustering of applications with noise (DBSCAN) clustering and ant colony optimization techniques.
\end{abstract}

\section{INTRODUCTION}

We propose an algorithm to plan survey and transit paths in order to re-acquire and identify a set of underwater objects distributed over a large area. The algorithm partitions the set of objects into separate clusters so that tightly-grouped objects are surveyed holistically where advantageous, and separately when appropriate. We demonstrate that the DBSCAN clustering algorithm chose to perform the partitioning has parameters that may be tuned using analytical techniques in order to incorporate unique characteristics of the underwater vehicle used for the inspection. Our work leverages the previously-developed algorithms to solve the multiple aspect coverage (MAC) problem for surveying multiple contacts simultaneously when the contacts are grouped closely together [1]-[2].

Maritime search and inspection missions for detecting and identifying underwater objects of interest are typically separated into two phases. The first phase involves surveying an area with a low-resolution, high coverage sensor that result in a relatively large number of detections, including false contacts. The number of false contacts increases significantly in areas with high amounts of clutter. The second phase, typically named the re-acquire and identify (RI) phase involves inspecting detections from multiple aspect angles in order to determine if the detection is a true object of interest or a false contact. The multiple angles allow for multiple views of a contact in order to determine if it is indeed an object of interest, or merely a false contact.

A. Sriraman is with the Sibley School of Mechanical \& Aerospace Engineering, Cornell University, Ithaca, NY 14850, as2587@cornell.edu

M. J. Bays is with the Naval Surface Warfare Center - Panama City Division, Panama City, FL 32407, matthew.bays@navy.mil

Distribution Statement A: Approved for public release; distribution is unlimited.
Matthew J. Bays

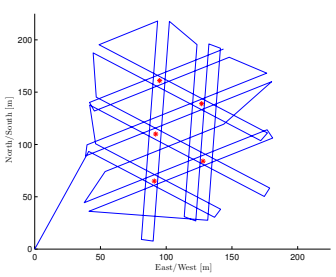

(a) MAC Algorithm

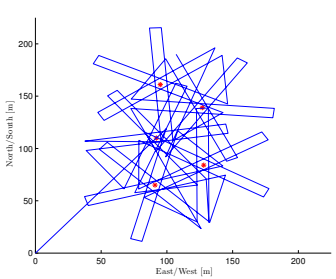

(b) Standard Algorithm
Fig. 1. An example of the using MAC with an effective cluster. The path generated by the MAC algorithm $(d=3280 \mathrm{~m})$ is $24 \%$ shorter than the standard path $(d=4330 \mathrm{~m})$

The algorithm discussed in [1]-[2], which we term the multiple-aspect coverage (MAC) algorithm, has been shown to be significantly more efficient than the standard, singlecontact pattern when the contacts occur in close proximity. However, when the contacts span a great distance, the algorithm often creates paths that are less efficient than using a single-contact pattern. The efficiencies and inefficiencies are illustrated in Figures 1 and 2: the MAC algorithm reduces the distance by $24 \%$ (compared to executing the standard, single-object pattern over each object) in a dense situation. However, the sparse distribution of the objects in the example causes the MAC algorithm to plan a path that is $18 \%$ longer than when executing the single-contact pattern over each contact. Sparser distribution examples can result in even poorer performance. We seek to implement a clustering algorithm that can partition a set of contacts into individual clusters that can then effectively use the MAC algorithm. Contacts may be distributed unevenly over an area, and may follow no pre-determined pattern of distribution; hence, there can be no lower or upper bound on the number of clusters to be created from the input data set. We consider a variety of clustering algorithms for the task: k-means, quality threshold (QT), and density-based spatial clustering of applications with noise (DBSCAN).

Execution of the MAC algorithm over each cluster generates a set of straight-line vehicle trajectories, termed swaths, that are used to collect sensor data, as well as transition segments connecting them. The current heuristic implemented in the MAC algorithm clusters the swaths such that swaths with close centroids are paired together. Upon clustering the swaths, a greedy traveling salesman problem (TSP) algorithm is used to order the traversal of swath-clusters. The greedy TSP method of path planning has proven to be inefficient, especially when the generated swaths require a significant amount of transit distance between swaths to the global 


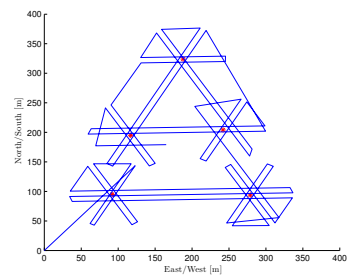

(a) MAC Algorithm

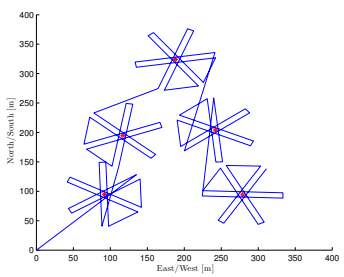

(b) Standard Algorithm
Fig. 2. An example of the using MAC with an ineffective cluster. The path generated by the MAC algorithm $(d=5410 \mathrm{~m})$ is $18 \%$ longer than the standard path $(d=4580 \mathrm{~m})$.

path. To solve the problem of connecting the swaths, we implement a two-tiered Ant-Colony optimization routine in order to first choose the order of shortest distance to transit for each partitioned cluster, and then to connect the swaths of a pattern developed using the MAC algorithm for the clusters.

This paper is outlined as follows: in Section II, we discuss the formal setup of the problem, shortcomings of previous algorithms, and our approach to increasing path efficiency. In Section III, we discuss our clustering solution in detail, including our techniques for parameter selection. We provide a brief overview of the MAC algorithm from previous research in IV. We propose a modified ant colony optimization (ACO) solution to the traveling salesman problem for connecting the survey paths for the overall path in Section $\mathrm{V}$. The performance of the modified algorithm is discussed in Section VI.

\section{Problem Definition}

We address an end-to-end path planner in order to perform a re-acquire and identify mission over $N$ contact locations $\chi_{i}=\left[x_{i}, y_{i}\right]^{\top} \in \mathbb{R}^{2}$ estimated by $\hat{\chi}_{i}=\left[\hat{x}_{i}, \hat{y}_{i}\right]^{\top} \in \mathbb{R}^{2}$ for $i=1, \ldots, N$ from a previous detection mission in an efficient manner. The error $\nu_{i}$ for $i=1, \ldots, N$ in the contact location estimates is assumed to be zero-mean, uncorrelated and Gaussian with covariance,

$$
E\left[\boldsymbol{\nu}_{i} \boldsymbol{\nu}_{i}^{\top}\right]=\left[\begin{array}{cc}
\left(\sigma_{i x}\right)^{2} & 0 \\
0 & \left(\sigma_{i y}\right)^{2}
\end{array}\right]
$$

The contact location estimates are provided in the vector

$$
\hat{\chi}=\left[\hat{\chi}_{1}^{\top}, \ldots, \hat{\chi}_{N}^{\top}\right]^{\top}
$$

\section{OBJect Clustering}

In the first stage in our large-area RI planning algorithm, we must partition a holistic list of possible underwater objects into efficient clusters. Clustering is an extremely effective tool to group similar data together; definitions of similarity help decide the appropriate clustering algorithm for a given application. In our application, similarity between two objects is directly related to the distance a unmanned underwater vehicle (UUV) must travel between them. In this section we first discuss the high-level clustering algorithm characteristics conducive to RI missions in the context of three common clustering algorithms, and outline how the characteristics lead to the selection of the DBSCAN clustering algorithm in order to partition an object list. We then propose a parameter selection methodology to tune the DBSCAN clustering algorithm based on a priori information about the UUV dynamics and object uncertainty.

\section{A. Clustering Method Comparison and Selection for RI Missions}

Clustering algorithms generally fall under two categories: partitional and hierarchical. Partitional clustering is used to divide the set of data objects into non-overlapping subsets. Hierarchical clustering creates a set of nested clusters organized as a tree. For the application of clustered RI prosecution, we are interested in a partitional clustering algorithm capable of dividing a set of input points into any number of non-overlapping subsets. Additionally, we require complete clustering - ensuring no points are disregarded as noise.

The most widely used partitional clustering method is $k$ means clustering [3]. $k$-means clustering partitions the data into $k$ clusters, each associated with a centroid. However, a setback of $k$-means clustering is its $k$ input parameter defining the number of clusters that has to be known a priori. Since the most efficient distribution of object points is unknown for each repetition, guessing the correct number of clusters becomes an impossible task and renders $k$-means clustering ineffectual for RI prosecution.

In order to understand QT and DBSCAN clustering algorithms, we first must define the following terms used in the context of the algorithms: $\varepsilon$-neighborhood of an object, directly density-reachable, and density-reachable.

Definition 1. ( $\varepsilon$-neighborhood of an object) The $\varepsilon$ neighborhood of an object, denoted by $\mathcal{N}_{\varepsilon}(\hat{\chi})$, is defined by $\mathcal{N}_{\varepsilon}(\hat{\boldsymbol{\chi}})=\left\{q \mid \operatorname{dist}_{\mathcal{L}_{2}}(\hat{\boldsymbol{\chi}}, q) \leq \varepsilon\right\}$

Definition 2. (directly density-reachable) A point is directly density-reachable from a point $q$ wrt. $\varepsilon$ if

1) $\hat{\chi} \in \mathcal{N}_{\varepsilon}(q)$ (core point condition) $\varepsilon$

Definition 3. (density-reachable) A point is densityreachable from an object $\hat{\chi}^{\prime}$ wrt. $\varepsilon$ if there is a chain of objects $\hat{\chi}_{1}, \ldots, \hat{\chi}_{N}$, such that $\hat{\chi}_{i+1}$ is directly densityreachable from $\hat{\chi}_{i}$.

Quality threshold (QT) clustering is another widely-used clustering method, where the points are clustered based on their proximity to a centroid instead of their proximity to each other [4]. In QT clustering, each point starts within its own individual cluster. If a point is directly density-reachable from that point, the two clusters are merged into a single cluster. As the measure that determines the clustering is the distance between a point in the cluster and an object, QT clustering will lead to a significant number of clusters when objects are uniformly positioned in long lines, even when the distance between objects is small.

Density-based spatial clustering of applications with noise (DBSCAN) is a popular clustering algorithm known for its ability to detect noise and cluster an oddly shaped set of 


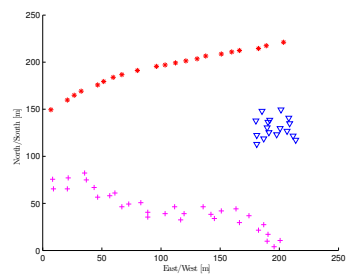

(a) DBSCAN

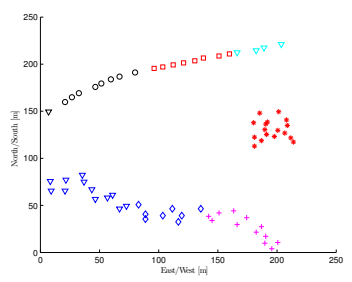

(b) QT Clustering
Fig. 3. An example of clustering using DBSCAN (obtaining three clusters) and QT Clustering (obtaining eight clusters).

points [5]. The algorithm has two tunable parameters: the minimum number of objects that can form a cluster $P_{\min }$ and the distance between two objects to be in the same cluster $\varepsilon$.

The DBSCAN and QT clustering algorithms follow a similar process. They both arbitrarily pick a point as a "core object" and search for points in a radius around the core object - this radius is referred to as $\varepsilon$ by DBSCAN and a "threshold" by QT. DBSCAN continues to find more points that are density-reachable by each of the points in the neighborhood of the original core object; this causes DBSCAN to have a linking-effect where a cluster can grow far from the initial core object and have odd shapes. QT clustering, however, only allows for direct density-reaching; each cluster generated by QT is contained within the threshold value of a core-object. The comparison between these algorithms can be seen in Figure 3. We chose DBSCAN as the final clustering algorithm for RI missions because of its ability to find long yet dense clusters. Regardless of the distribution of points, the algorithm always returns clusters containing points closely grouped together and density-connected.

\section{B. DBSCAN Clustering Parameter Selection}

As in any clustering algorithm, the effectiveness of DBSCAN for use in partitioning object lists for RI missions is greatly dependent on the values of it's tuning parameters. We now turn to selecting the parameters for the DBSCAN clustering algorithm: $P_{\min }$ and $\varepsilon$. We first note that $P_{\min }$ is a parameter to determine the minimum number of points required for a cluster to exist. If the number of objects in a cluster is less than $P_{\text {min }}$, the objects are considered noise, and not part of the resulting dataset. Since ignoring a group of objects, no matter how small, is fundamentally in conflict with the nature of an RI mission, using the DBSCAN clustering method for RI missions requires

$$
P_{\text {min }}=1 \text {. }
$$

An appropriate value for the parameter $\varepsilon$ is less trivial. As discussed earlier, $\varepsilon$ is the maximum distance from one object to another object for the two objects to be in the same cluster. If the distance is large, the few clusters will be generated and there will be a greater distance between objects in the clusters. If $\varepsilon$ is small, many clusters of tightlygrouped objects will be generated. Because the distribution of objects in an area is unknown at the start of a mission, the distribution of the objects cannot be used to determine the parameter set.

Additionally, the primary drivers of the efficiency of an RI pattern over objects prosecuted individually versus holistically is not their distance alone, but their distance as related to the required track length for an individual pattern. Figure 4 illustrates the concept of object clustering and travel savings related to required travel distances. The figure shows two objects with notional star patterns overlayed on them. An RI track nominally consists of two track lengths: the steady-state length, $L_{s s}$, and the uncertainty radius, $R_{u}$. The length $L_{s s}$ is the additional length a track needs in one direction in order to account for stabilizing the vehicle from any turning motions, distance required by the sonar in order to start providing meaningful data, or other issues not related to the object. The value of $L_{s s}$ is vehicle-dependent, and is typically a constant found through modeling or sea testing. The distance $R_{u}$ is the radius that represents the possible location of an object based on the object's uncertainty.

In order to find the distance $R_{u}$, we must have the required along-track probability of surveying the object $\mathcal{P}_{\text {track }}$ and object variance from (1). The value of $\mathcal{P}_{\text {track }}$ is typically found precisely as part of the MAC algorithm, as a function of the desired probability of re-acquiring all objects $\mathcal{P}$ and the number of tracks required to survey the cluster. However, from numerical methods show a conservative value is $\mathcal{P}_{\text {track }}=0.98$ for $\mathcal{P}=0.85$. From $\mathcal{P}_{\text {track }}$ and (1), may find a conservative one-dimensional radius required to survey a object as

$$
R_{u}=\sqrt{2} \operatorname{erf}^{-1}\left(2 \mathcal{P}_{\text {track }}-1\right)_{\{i=1, \ldots, N\}, j=\{0,1\}} \sigma_{i j}
$$

where $\operatorname{erf}^{-1}(\cdot)$ is the inverse error function. Equation (4) finds the shortest distance that will insure crossing all objects within the specified probability of coverage in the along-track direction.

Because tracks may be followed in either direction, and often the end of one track leads into the beginning of another track, the distances are required on either side of nominal single-object RI patterns. However, when objects are grouped, tracks that will survey both objects do not need track lengths for the vehicle to reach steady-state and cover the uncertainty radius, so long as the objects are close together. On the other hand, if the objects are grouped but far enough apart so that the required distances do not overlap, more distance is required in order to create a single track. Our goal is to determine an appropriate clustering parameter $\varepsilon$ as a function of $R_{u}$ and $L_{s s}$ such that when objects are close enough to together, the objects are clustered together. Otherwise, the parameter should cause the objects to be partitioned into separate clusters.

From $L_{s s}$ and (4), we can find the threshold where grouping two objects will be generally as efficient as prosecuting them separately,

$$
\varepsilon_{\max }=2\left(R_{u}+L_{s s}\right) .
$$

If $\varepsilon$ is less than the value in (5), travel distance will be saved. We also know that if $\varepsilon=0$, the clustering algorithm 


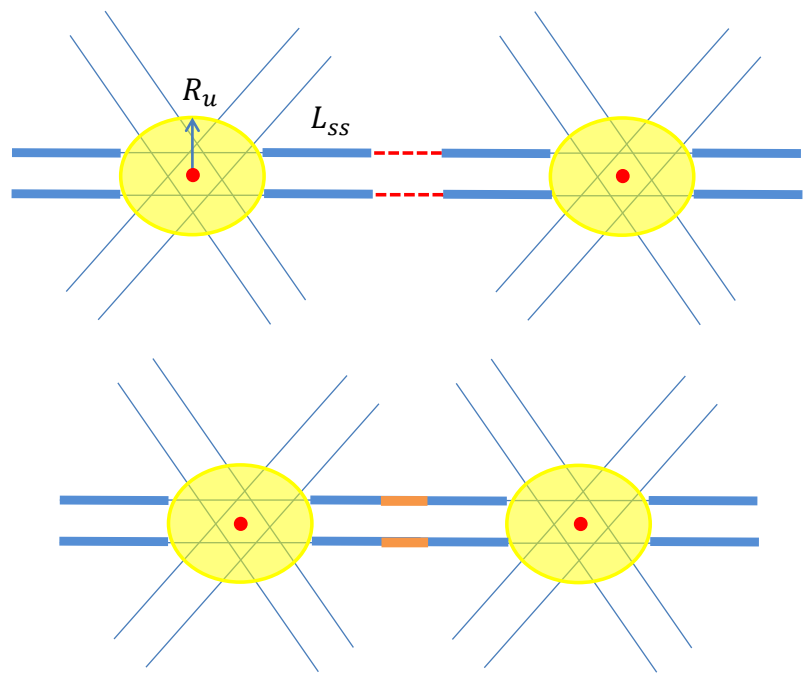

Fig. 4. An illustration of required RI transit distance. Yellow circles indicate object location error ellipses. Blue thick lines indicate required transit lengths for steady state of sensor. Top: Instance where RI path would not benefit from prosecuting objects together. Red dashed lines indicate wasted distance if horizontal tracks were combined. Bottom: Instance where horizontal tracks would benefit from combining object prosecution. Orange thick lines indicate potential overlap and savings if tracks were combined.

will yield the same result as prosecuting objects individually, since no objects will be density-reachable. Increasing the value from $\varepsilon=0$ will yield larger clusters and potential savings in travel distance, thus

$$
0 \leq \varepsilon_{\text {opt }} \leq \varepsilon_{\max }
$$

Assuming that the effectiveness of the clustering between $\varepsilon=0$ and $\varepsilon_{\max }$ follows a parabolic path, a reasonable parameter choice would then be

$$
\varepsilon_{\text {opt }} \approx\left(R_{u}+L_{s s}\right) .
$$

Figure 5 shows a plot of $\varepsilon$ versus the average ratio of the transit distance for clustering RI patterns to the same lay-down

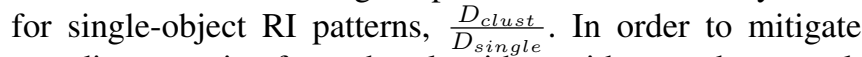
any discrepencies from the algorithm with regards to track choice or track length of a standard pattern versus the MAC technique, the proposed algorithm was run over every object individually to calculate $D_{\text {single }}$. As shown in the figure, the ratio generally decreases, implying greater efficiency for the clustered RI approach, until $\varepsilon=87$. We note that the predicted value of $\varepsilon_{\text {opt }}$ is not the global minumum from the simulation, but very close: $\frac{D_{\text {clust }}}{D_{\text {single }}}=0.7666$ vs. 0.7642 . After $\varepsilon_{o p t}$ is reached, the ratio starts increasing until $\varepsilon_{\max }$. Furthermore, $\frac{D_{\text {clust }}}{D_{\text {single }}}$ generally increases after $\varepsilon_{\text {opt }}$ up to $\varepsilon_{\max }$.

Thus, we have the parameter selection of (3) and (7) for our chosen clustering method. We next provide a brief overview of the Multiple Aspect Coverage algorithm, and the modifications made in order to combine the clustering and TSP solver with previous research.

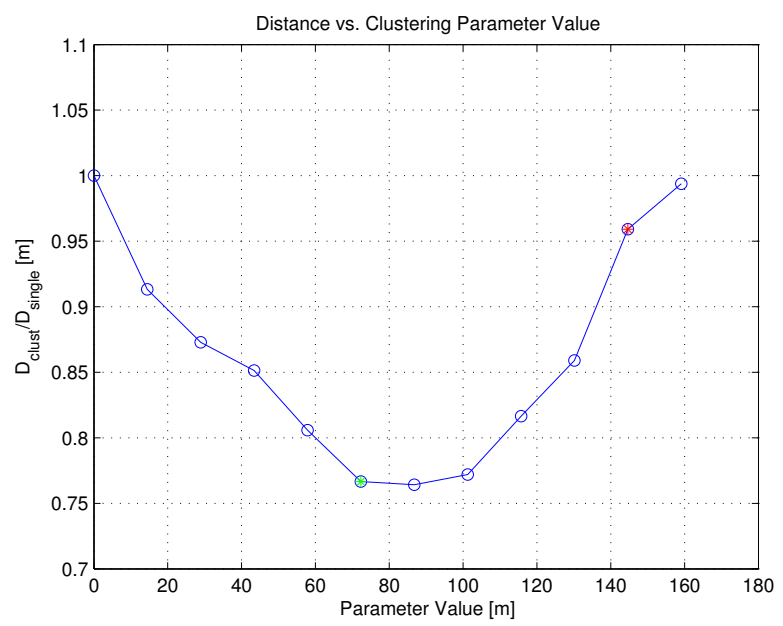

Fig. 5. Plot of effect of varying DBSCAN Parameter $\varepsilon$ to change in the distance ratio $\frac{D_{\text {clust }}}{D_{\text {single }}}$. Red $*$ indicates value of $\varepsilon_{\max }$. Green $*$ indicates predicted value of $\varepsilon_{\text {opt }}$. A ratio below one indicates that using the indicated parameter value causes the algorithm to out-perform the standard, singleobject technique.

\section{BRIEF OVERVIEW OF THE MULTIPLE ASPECT COVERAGE ALGORITHM}

Once the objects are partitioned into smaller clusters, each cluster is consecutively processed using a modified version of the Multiple Aspect Coverage (MAC) algorithm found in [1]-[2]. We will now provide a high-level overview of the algorithm as well as details of its current modifications. For an individual cluster of objects, the MAC algorithm finds the best set of global directions, also known as aspects, from which to survey every object. The optimal aspect is found by creating a convex hull of the objects, and calculating a function related to the width of the convex hull in the direction of each side. The side that produces the global minimum of the objective function is the optimal direction of the first aspect, with the other aspects constrained to be at equal angular spacing from the first aspect. The method used to find the aspects has been proven through analysis to be optimal for uniform coverage assumptions.

Once the set of aspects is determined for a cluster, the algorithm plans the track locations for each aspect. The position is found by first creating a probability density in the form of a Gaussian mixture model (GMM) of the expected object locations relative to a chosen aspect. The algorithm then weights the GMM by the sensor profile and integrates for all candidate positions within the aspect; this is equivalent to the expected probability of detection.

The algorithm selects the location of each track based on the global maximum of the objective function. Once a track is selected, the GMM is updated to incorporate the information expected to be gained from the survey, and the objective function is re-calculated to select the next track in an aspect. In this manner previous tracks for the same aspect reduce the score of the objective function at their location, thus increasing the likelihood that tracks at different locations 
will be selected. The algorithm continues to select tracks in a given aspect until a threshold is reached. The algorithm uses the GMM as a formal upper-bound on the probability of successfully surveying every object from every aspect. This upper-bound is compared to a user-specified threshold. An additional improvement was achieved by modifying the threshold probability calculations used by each aspect based on current MCM theory. We have modified the algorithm such that the output is now the set of swaths $\mathbf{S}$. For additional information on the original algorithm, we refer to [1]-[2]. Using the MAC algorithm for choosing the swaths within each cluster, we next turn to planning the transit paths and ordering of the swaths in between each cluster.

\section{MODIFIED ANT COLONY OPTIMIZATION FOR SURVEY SEGMENTS}

Once the algorithm selects a sufficient number of tracks in every aspect to meet the overall probability of success, the tracks are sent to a low-level ACO TSP solver to connect the swaths used to prosecute the cluster. We used a modified algorithm similar to the ACO TSP solver found in [6]. The ACO TSP solver plans a path from the current UUV position, the set of swaths required to survey the current cluster, and a specified endpoint. The endpoint is set as the centroid of the next cluster from the higher-level TSP solver. We define the set of waypoints $\mathbf{W}$ for the TSP optimization as all of the waypoints from the start, end, and set of swaths S. While traditional TSP solvers optimize transits between individual points of $\mathbf{W}$, the low-level TSP solver imposes constraints forcing the routine to execute waypoints comprising a survey track consecutively. This means that one waypoint that comprises a track pre-determines the next waypoint automatically, but factors the length of tracks and inter-track distances into the optimization. The constraints are necessary so that the required tracks are executed while optimizing their order for the minimum distance the UUV must travel. An ancillary benefit is that the optimization space of the TSP algorithm is reduced significantly, decreasing the computational complexity and time required to find a solution.

Initially, $N_{\text {ants }}$ agents are placed on randomly selected waypoints. At each time step the agents move to different waypoints and increase the travel reward $\tau\left(w_{1}, w_{2}\right)$ on the segment $\left(w_{1}, w_{2}\right)$ of each agent's traveled path. The agent at waypoint $w_{1}$ chooses the next waypoint $w_{2}$ using a probabilistic function both of trail accumulated on the swaths or connecting transit paths $\tau\left(w_{1}, w_{2}\right)$ and of a heuristic function of path length between the two waypoints $\eta\left(w_{1}, w_{2}\right)$. For our system,

$$
\eta\left(w_{1}, w_{2}\right)=\frac{1}{\operatorname{dist}\left(w_{1}, w_{2}\right)}
$$

The probability that an agent would pick $w_{2}$ as the next waypoint to visit is

$p\left(w_{1}, w_{2}\right)=\left\{\begin{array}{cc}1 & \text { If }\left(w_{1}, w_{2}\right) \in \mathbf{S} \\ \frac{\left[\tau\left(w_{1}, w_{2}\right)\right] \cdot\left[\eta\left(w_{1}, w_{2}\right)\right]^{\beta}}{\sum_{w \in W}\left[\tau\left(w_{1}, w\right)\right] \cdot\left[\eta\left(w_{1}, w\right)\right]^{\beta}} & \text { If } w_{2} \in J\left(w_{1}\right) \\ 0 & \text { Otherwise }\end{array}\right.$

where $J\left(w_{1}\right)$ is the set of waypoints that have not been visited by the agent positioned at $w_{1}$, and $\beta$ is a parameter which determines the relative importance of the trail function. Once every agent has toured all of the waypoints, the trail for each segment is updated using

$$
\tau\left(w_{1}, w_{2}\right) \leftarrow(1-\alpha) \cdot \tau\left(w_{1}, w_{2}\right)+\sum_{k=1}^{N_{\text {agents }}} \Delta \tau_{k}\left(w_{1}, w_{2}\right)
$$

where

$\Delta \tau\left(w_{1}, w_{2}\right)=\left\{\begin{array}{cc}\frac{1}{\operatorname{dist}\left(w_{1}, w_{2}\right)} & \text { If }\left(w_{1}, w_{2}\right) \in \text { tour of agent } k \\ 0 & \text { Otherwise }\end{array}\right.$

and $\alpha$ is a travel reward decay parameter.

Using (8)-(11), agents probabilistically choose nearby waypoints that are connected by paths traveled by previous agents. Once all the agents have completed the entire path the agent that transited using the least travel distance modifies the paths belonging to its plan by adding an additional travel reward that is inversely proportional to the tour length. After the algorithm converges to a specified threshold between the minimum and maximum distance an agent travels to transit all swaths $\varepsilon_{a n t s}$, or the maximum number $T_{\max }$ of iterations occur, the algorithm ends and the shortest path is output as the final path.

\section{SIMULATION RESULTS}

We have simulated the proposed algorithm in order to compare it with both the original MAC algorithm and the standard, single-object pattern typically used for RI missions by the UUV community. We used a clustering parameter from (7) of $\varepsilon_{o p t}=72 \mathrm{~m}$. For the ACO algorithm, we used $\alpha=1$, and $\beta=5$. The ACO algorithm used $N_{\text {agents }}=$ 400 agents and $T_{\max }=60$ iterations. Figure 6 shows a comparison between a set of objects executed using the previously developed MAC algorithm alone, and the new algorithm using efficient clustering and ACO TSP solvers. As seen in the figure, the original MAC algorithm creates a number of inefficient tracks when objects are far apart but prosecuted simultaneously. The overall distance required by the original algorithm was $7194 \mathrm{~m}$, while the modified algorithm required only $4975 \mathrm{~m}$.

Figure 7 shows the results of a Monte Carlo simulation of the proposed algorithm on a $926 \mathrm{~m}$ x $926 \mathrm{~m}$ (1/4 square nautical mile) field with 20 randomly placed objects. The algorithm was compared against a RI pattern commonly used consisting of three aspects with four tracks per aspect to identify an object with a mean-squared position error of $15 \mathrm{~m}$. The probability of success for the proposed algorithm was set to $\mathcal{P}=0.85$, with $\mathcal{P}_{\text {track }}=0.98$. As shown in the figure, the use of the advanced RI planning algorithms yields significant 


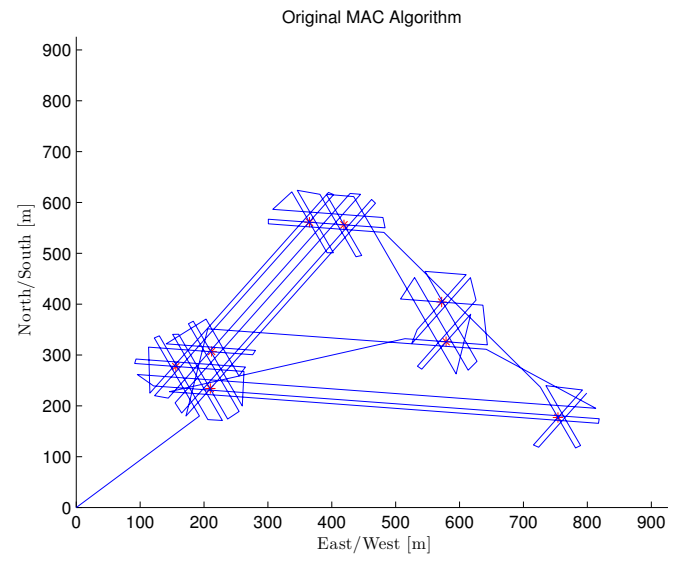

(a) Original MAC Algorithm

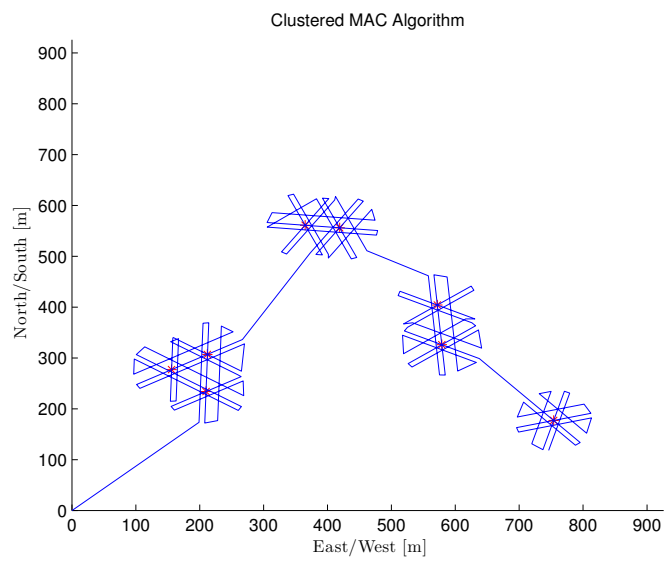

(b) Proposed Algorithm

Fig. 6. Left: A cluster of eight objects prosecuted by the basic MAC algorithm from [1]-[2]. Right: Proposed algorithm combining DBSCAN clustering and an ACO TSP algorithm. [1]

travel savings for the UUV, potentially as high as $41 \%$ of the distance required by standard techniques. Additionally, the algorithm significantly reduces the number of turns required by an average of $39 \%$. The reduction in travel distance and vehicle turns will significantly reduce mission time. Part of the travel and turn savings are due to the advanced algorithms prosecuting objects holistically. Additional travel savings are due to the standard pattern being overly conservative for the object position error and assumed. When the contact density is higher or there is greater certainty of object positions, the travel savings will be greater due to the algorithm taking the more favorable characteristics into account when developing the RI paths.

Figure 8 shows the results of a simulation of a real-time implementation of the algorithm within the Mission-Oriented Operating Suite - Interval Programming (MOOS-IvP) autonomy framework. A UUV is tasked with prosecuting two clusters of targets. The module consists of a clustering and queuing module, and a slightly modified version of the original MAC algorithm. Future experiments are planned to demonstrate algorithm performance on UUVs in the field.

\section{CONCLUSION}

We have presented an end-to-end solution to plan a vehicle path for re-acquiring and identifying underwater objects over large areas. The solution uses the DBSCAN clustering algorithm to partition the object list into efficient segments, with the values of the clustering parameters chosen from mission requirements and vehicle dynamics. The solution also leverages previous work to develop the vehicle survey tracks, and optimizes the transits between tracks using an ant colony optimization technique modified for optimizing RI tracks. Ongoing work involves allowing for varying object location uncertainties, as well as incorporating real-time estimated vehicle GPS error as an additional input.

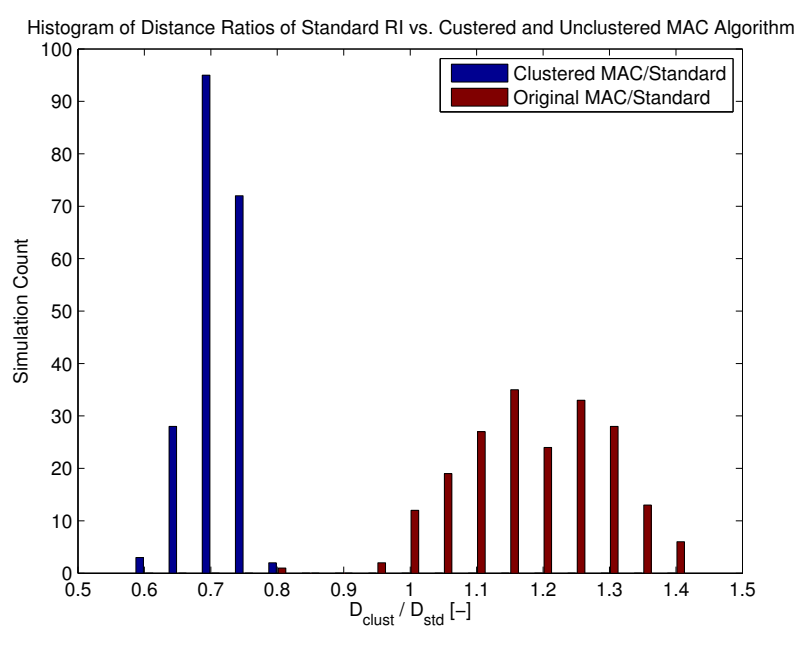

Fig. 7. Histogram of 200 simulations of the MAC algorithm when compared to the standard single-object technique. Blue bars indicate modified algorithm including the DBSCAN clustering step and ACO Optimization. Red bars indicate use of original MAC algorithm. In each simulation twenty objects were randomly placed on a $926 \mathrm{~m}$ x $926 \mathrm{~m}$ (1/2 nautical mile $\mathrm{x}$ $1 / 2$ nautical mile) field. The use of the algorithm produced an average of $29 \%$ reduction in distance traveled when compared to standard RI pattern. Histogram shows the ratio of the clustered, TSP-integrated MAC technique divided by the distance required by the standard, single object technique for the same objects. A ratio below one indicates the proposed technique out-performs the standard, single-object technique.

\section{ACKNOWLEDGMENTS}

The authors gratefully acknowledge the discussions with Dr. John Hyland and Dr. Greg Garcia of the Naval Surface Warfare Center Panama City Division on various aspects of this work.

\section{REFERENCES}

[1] M. J. Bays, A. Shende, D. J. Stilwell, and S. A. Redfield, "Theory and experimental results for the multiple-aspect coverage problem," Ocean Engineering, vol. 54, 2012.

[2] - "A solution to the multiple aspect coverage problem," in International Conference on Robotics \& Automation, 2011. 


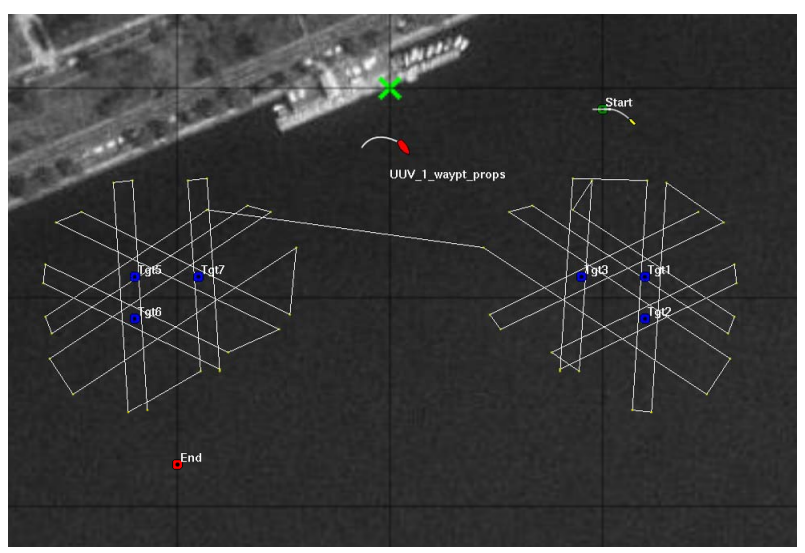

Fig. 8. Image of MOOS-IvP simulation with improved MAC algorithm.

[3] J. MacQueen, "Some methods for classification and analysis of multivariate observations," in Proceedings of the Fifth Berkeley Symposium on Mathimatical Statistics and Probability, 1967.

[4] L. J. Heyer, S. Kruglyak, and S. Yooseph, "Exploring expression data: Identification and analysis of coexpressed genes," Genome Research, vol. 9, pp. 1106-1115, 1999.

[5] M. Ester, H. P. Kreigel, J. Sander, and X. Xu, "A density-based algorithm for discovering clusters," in Proceedings of the Association for the Advancement of Artificial Intelligence, 1996.

[6] M. Dorigo, "Ant colony system: A cooperative learning approach to the traveling salesman problem," IEEE Transactions on Evolutionary Computation, vol. 1, pp. 53-66, 1997. 